\title{
Evaluation of Sentinel Lymph Node Biopsy and Axillary Lymph Node Dissection for Breast Cancer Treatment Concepts - a Retrospective Study of 1,214 Breast Cancer Patients
}

Roland G. Stein ${ }^{a}$ Roland Fricker ${ }^{b}$ Thomas Rink ${ }^{c}$ Hartmut Fitz $^{d}$ Sebastian Blasius Joachim Diessner $^{a}$ Sebastian F.M. Häusler ${ }^{a}$ Tanja N. Stüber ${ }^{a}$ Victoria Andreas $^{a}$ Achim Wöckel ${ }^{a}$ Thomas Müller ${ }^{b}$

a Department of Obstetrics and Gynecology, Würzburg University Hospital, Würzburg, Germany;

${ }^{b}$ Department of Obstetrics and Gynecology, Hanau City Hospital, Hanau, Germany;

${ }^{c}$ Institute for Nuclear Medicine, Hanau City Hospital, Hanau, Germany;

${ }^{d}$ Institute for Pathology, Hanau City Hospital, Hanau, Germany

\section{Keywords}

Breast cancer - Sentinel node biopsy .

Axillary lymph node dissection - Chemotherapy .

Axilla treatment . Supraclavicular fossa radiation

\section{Summary}

Background: Most breast cancer patients require lumpectomy with axillary sentinel lymph node biopsy (SLNB) or axillary lymph node dissection (ALND). The ACOSOG Z0011-trial failed to detect significant effects of ALND on disease-free and overall survival among patients with limited sentinel lymph node (SLN) metastases. Intense dose-dense chemotherapy and supraclavicular fossa radiation (SFR) are indicated for patients with extensive axillary metastases. In this multicentered study, we investigated the relevance of ALND after positive SLNB to determine adequate adjuvant therapy. Methods: We retrospectively analyzed data from 1,214 patients with clinically nodal negative T1-T2 invasive breast cancer undergoing surgery at Hanau City Hospital Breast cancer center. Results: 681 patients underwent ALND after SLNB. 20 patients (8.5\%) from the group with 1 or 2 SLN metastases $(n=236)$ showed more than 3 lymph node metastases after ALND. 13 patients (31.7\%) from the group with more than 2 SLN metastases ( $n=41$ ) were diagnosed with a minimum of 4 axillary

Roland Gregor Stein and Roland Fricker contributed equally to the publication lymph node metastases after ALND. Conclusions: In $8.5 \%$ of the patients with 1 or 2 SLN metastases, ALND detected more than 3 macrometastases, setting the indication for intense dose-dense chemotherapy and SFR. More than 2 SLN metastases, T stage and grading predict lymph node metastases.

(c) 2017 S. Karger GmbH, Freiburg

\section{Introduction}

Breast cancer is the most common cancer in women, with an incidence of about 70,000 cases in Germany per year [1]. The invasive carcinoma of no special type (NST) accounts for about 70$80 \%$ of breast cancers. Lobular invasive cancers are less common, comprising only $10-15 \%$ of all breast cancers [2]. In most cases with clinically unsuspicious axillary lymph nodes, surgery consists of lumpectomy with axillary sentinel lymph node (SLN) biopsy (SLNB). Due to the strong negative prognostic value of axillary lymph node metastases, axillary lymph node dissection (ALND) used to be performed in patients with clinical lymph node metastases as well as positive SLNB. The rate of ALND decreased over the last decade with persisting variance in clinical practice [3]. The German S3 guideline for breast cancer diagnosis and treatment recommends SLNB for initial axillary operative staging. ALND should be performed in patients with no detectable SLN and in cases with SLN macrometastases [4].

\section{KARGER}

() 2017 S. Karger GmbH, Freiburg
Roland Gregor Stein, MD 
The prospective randomized controlled ACOSOG Z0011 trial changed the view towards ALND [5]. Giuliano and colleagues compared the outcome of patients with T1-T2 breast cancer with or without ALND after detection of SLN metastases and did not find a significant difference in terms of disease-free survival, overall survival and local recurrence of breast cancer. Although the number of patients in the study was not sufficient for statistical significance of that result, it brought forth a discussion on a paradigm shift in breast cancer surgery. Other studies such as the NSABP B04 trial corroborated this result, finding no impact of ALND on overall survival [6]. Even for the subgroup of patients with 3 or more lymph node metastases, no significant difference could be found in terms of overall or disease-free survival in a retrospective data analysis [7]. Rudenstamm et al. [8] compared clinically nodal negative patients with and without ALND and found no difference regarding cancer recurrence and overall patient survival between these 2 groups. Only 1 meta-analysis comprising studies without adjuvant systemic therapy found a $5.4 \%$ improvement in overall survival in the ALND subgroup [9].

Since ALND, compared to SLNB alone, increases postoperative morbidity - such as arm swelling or numbness [10-12], axillary surgery should be reduced to the required minimum [13]. Solid tumor treatment generally adapts to the idea of cancer as a cellular disease. According to the cancer stem cell hypothesis, a small subgroup of cancer cells is responsible for metastases and relapse, due to their ability to self-renew and form new tumors [14]. This suggests that the outcome of breast cancer mostly depends on cancer cell characteristics, instead of depending on the potential leftover residual disease, i.e. in the axilla before adjuvant systemic therapy. Nonetheless, the number of axillary lymph node metastases is relevant for the staging of breast cancer lymph node status. A randomized controlled trial of intense dose-dense systemic therapy published by Moebus and colleagues $[15,16]$ significantly improved both disease-free and overall survival in patients with 4 or more positive axillary lymph nodes. This proved the tendency already found in the CALGB-9741 trial that dose-dense chemotherapy is more effective than conventional regimens [17].

Axillary lymph node status also impacts adjuvant radiotherapy as a part of breast-conserving surgery. Patients with 4 or more axillary lymph node metastases are recommended to undergo a supraclavicular fossa radiotherapy to reduce local recurrence $[18,19]$. The Early Breast Cancer Trialists' Collaborative Group (EBCTCG) had reported that adjuvant radiation of the chest wall, the supraclavicular region and/or the axilla after mastectomy and axillary surgery significantly reduced the rate of recurrence and mortality [20].

In this multicentered health services research approach, we investigated the axillary lymph node status of breast cancer patients after positive SLNB during initial surgery. The underlying goal was to determine the proportion of patients with an indication for intense dose-dense chemotherapy that would have remained undetected without ALND. We also evaluated the recommendations of the German S3 Guideline concerning ALND in our multicentered patient collective. Since the cited data suggest a less extensive axil- lary surgery for the future, we sought to determine histopathological characteristics that potentially support the adjuvant therapy indication.

\section{Material and Methods}

The study was performed at Hanau City Hospital, Germany. In this multicentered research approach, we retrospectively analyzed data from 1,214 patients diagnosed with T1-T2 invasive breast cancer undergoing primary surgery at Hanau City Hospital Breast Cancer Center and 2 other cooperating German clinics of obstetrics and gynecology (Hanau City Hospital ( $\mathrm{n}=880$ ); MainKinzig-Hospital, Gelnhausen ( $\mathrm{n}=226)$; St. Vinzenz Hospital, Hanau $(\mathrm{n}=168)$ between 1998 and 2011. The SLNB was evaluated and established at Hanau City Hospital [21] and has rapidly become part of daily routine. Rink et al. [22, 23] established standardized protocols for SLN detection and found that scintigraphical visualization of SLNs is influenced by metastatic involvement.

Inclusion criteria for the study were a primary diagnosis of unilateral breast cancer with an unsuspicious nodal status in clinical examination and sonogram and a clinical indication for primary breast cancer surgery with SLNB followed by ALND. Patients with an indication for neoadjuvant chemotherapy were excluded.

The SLNBs were executed according to a 2-day protocol, with tracer-application on day 1 and additional intraoperative Patent Blue application on day 2. The surgery was performed by gynecological breast surgeons in 1 of the 3 clinics. The tracer applications and the histopathological examinations were performed at Klinikum Hanau in the departments of nuclear medicine and pathology, respectively. The SLNs were evaluated on 2-mm sections with hematoxylin/eosin staining (H\&E); immunohistochemistry (IHC) was performed until 2004. The respective protocols have already been published [21-23].

According to the intraoperative pathological examination, SLN metastases led to ALND (as well as a not detectable sentinel node), with the excision of a minimum of 10 axillary lymph nodes. Since data assessment started in 1998, when SLNB was not a standard procedure, axillary surgical staging was performed by ALND and SLNB in 404 patients even after negative SLNB. ALND was performed only after informed patient agreement in cases with SLN micrometastases. Metastases thus comprised both micro- and macrometastases. The finding of isolated tumor cells (i+) did not lead to ALND. Tumor stage was defined according to the postoperative histopathological examination.

In the statistical analysis, frequencies were calculated for categorical variables. For continuous variables, mean, median, standard deviation, minimum and maximum were reported. The correlation of 2 categories was analyzed using cross-tables and Chi-square-test or Fisher's exact test (for $2 \times 2$ cross-tables). Differences between a continuous and normally distributed variable in more than 2 groups were calculated by ANOVA with Welch adaptation in case of non-equality. Kruskal-Wallis test was used for non-normally distributed data. The t-test was utilized for calculating differences in 2 groups in continuous and normally distributed variables, in case of non-equality with the help of the Welch adaptation. Logistic regression models were used to investigate the effect on binary outcomes. The effect of each factor was interpreted via odds ratio and $\mathrm{p}$ values. A significance level of $5 \%$ was used. All statistical analyses were conducted with SPSS version 22 (IBM, Armonk, USA).

The data for this study were acquired for quality assurance. The local ethics committee had no objections to the retrospective analysis of such information.

\section{Results}

Of the 1,214 patients with T1-T2 primary breast cancer screened, 851 showed T1 tumors, and 363 showed T2 breast cancers. Patients' age was between 27 and 92 years with a mean age of 61.83 years (SD 12.055) and did not differ between the 3 clinics 
Fig. 1. We present a nomogram for the subgroup of patients with 0-2 positive sentinel lymph nodes (SLNs). Taking into account all individual patient data and summing up the respective points gives a total points score that allows the likelihood of further positive lymph nodes to be predicted.

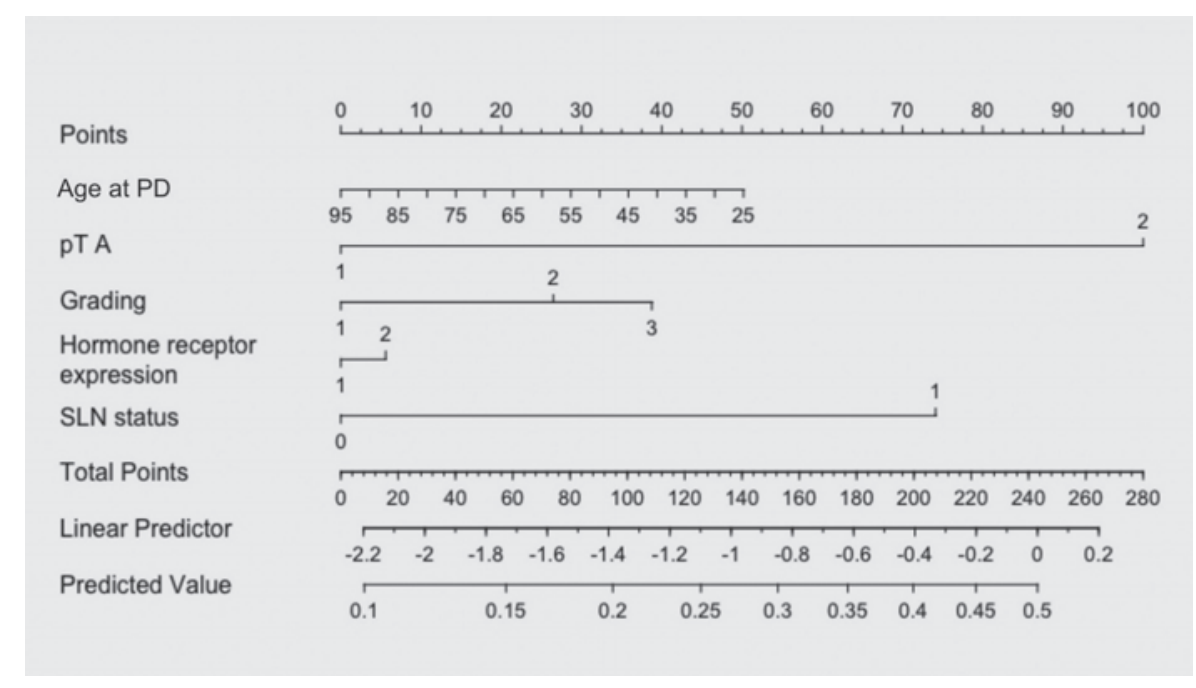

( $\mathrm{p}=0.058)$. A detectable SN was found in 1,158 patients $(95.4 \%)$; 822 in those with T1 tumors and 336 in those with T2 breast cancers. 112 tumors were graded as G1 (9.7\%, 112/1,158), 785 (67.8\%) as G2 and 261 tumors (22.5\%) as G3.

No SLN was detected in 56 cases. A mean of 2.6 SLNs were excised (SD 1.846). If ALND was performed, 11.04 (SD 8.525) lymph nodes were excised. This led to an overall number of excised lymph nodes of 14.02 (SD 8.938). 865 (74.7\%, 865/1,158) patients showed a negative SLN status, whereas 293 patients $(25.3 \%)$ were diagnosed with SLN metastasis (including 64 (5.5\%) cases with 1 to a maximum of 3 lymph nodes with micrometastases). In cases with macrometastases $(n=229)$, a mean of 1.73 positive lymph nodes were excised (SD 1.099) during SLNB.

Age significantly correlated with axillary lymph node metastases: Patients without axillary lymph node metastases had a mean age of 62.05 years (SD 11.766), while patients with axillary lymph node metastases had a mean age of 60.37 years (SD 12.850) $(\mathrm{p}=0.049)$.

We analyzed the correlation of the histopathological TNM tumor stage (pT1-pT2) and SLN metastases. As expected, an increasing tumor size, corresponding with higher $\mathrm{T}$ stage, significantly correlated with lymph node metastases in both SLNB and ALND ( $\mathrm{p}<0.001)$. Higher tumor cell grading showed a highly significant/significant correlation to lymph node metastases in both SLNB and ALND ( $\mathrm{p}=0.008 / \mathrm{p}=0.048)$.

Lymphangiosis carcinomatosa in primary tumors was associated with axillary lymph node metastases $(\mathrm{p}<0.001$, data not shown). In contrast, neither estrogen nor progesterone receptor expression correlated with axillary metastatic disease regardless of the respective tumor stage.

ALND was performed in 277 cases of SLN metastasis detection and in 404 cases even after negative SLNB, resulting in 681 patients recieving ALND after SLNB. 56 patients underwent ALND because no SLN was detectable making a total number of 737 patients with ALND. 95.2\% (648/681) of patients showed a maximum of 3 axillary lymph node metastases. Thus, the overall number of positive lymph nodes was 1.38 (minimum 0, maximum 33, SD 3.697).
In the group with 1 or 2 metastases in SLNB, 20 patients (8.5\%) showed more than 3 axillary lymph node metastases. In the group with more than 2 metastases in SLNB, 13 cases $(31.7 \%, 13 / 41)$ showed more than 3 axillary lymph node metastases. Alltogether, 33 patients (4.8\% of the 681 patients who underwent ALND after SLNB) were diagnosed with more than 3 axillary lymph node metastases. We sought to determine the likelihood of further lymph node metastases from SLNB results and individual patient data as well as histopathological information. The respective nomogram for the subgroup with 0-2 metastases in SLNB is presented in figure 1, from which the likelihood of further lymph node metastases according to the patients' individual characteristics can be calculated.

\section{Discussion}

Axillary surgery in clinically node-negative, early-stage breast cancer is still hotly debated after publication of the ACOSOG Z0011 trial data $[5,24]$. Of the 1,214 patients with primary T1-T2 breast cancer included in our study, 737 underwent ALND, 681 of them after SLNB. Since the data also included those for patients with ALND without positive SLNB, this study provides a special focus on the effects of omitting ALND for adjuvant treatment.

Our data collection started in the soon after the establishment of SLNB [21]. Since that time, numerous randomized controlled multicenter trials confirmed the performance and precision of SLNB as a surgical axillary staging procedure. This enabled the routine clinical application of the technique $[25,26]$. The SLN identification rate ranged from $95 \%$ to $98.7 \%$. The detection rate in our study (95.4\%) thus lies within this range.

We found a SLN-positive rate of $25.3 \%$ (including $5.5 \%$ cases with micrometastases). Other groups reported lower rates of between 13\% [27] and 17.6\% [28]; however, another German group recently published a comparable SLN-positive rate of $21.1 \%$ [29]. The rate of positive SLN is expected to decrease in the era of breast cancer screening. Therefore, not only the role of ALND but also 
that of SLNB itself is under discussion. Increasingly precise and efficient preoperative axillary imaging still misses about $25 \%$ of axillary metastatic disease - especially in cases with metastases of $\leq 5 \mathrm{~mm}$ and thus below the level of detection [29]. Wang et al. [30] recently published a meta-analysis that not only reported SLNB false-negative rates of up to $22.9 \%$, but also failed to find significantly different rates in overall survival, disease-free survival and local axillary recurrence with SLNB alone versus ALND. Zetterlund and colleagues [31] found axillary metastases after neoadjuvant chemotherapy in $7.4 \%$ of patients with negative SLNB before chemotherapy, giving a hint to potential false-negative SLNB. A recent meta-analysis revealed an overall SLNB false-negative rate of only $8.61 \%$ [32].

Four prospective trials randomized breast cancer patients with clinically unsuspicious axilla to standard axillary surgery versus no axillary surgery. These studies, however, showed a low overall rate of axillary recurrence in both groups, although axillary lymph node involvement was detected in $23-40 \%$ of the cases (in the ALND group). No significant differences were detected in terms of disease-free survival and overall survival [24]. These data indicate that omitting axillary surgery in patients with clinically and also diagnostically unsuspicious axilla seems to be a safe option. Further studies are needed to predict potential patient subgroups profiting from axillary surgery.

However, in our group of patients with 1-2 SLN metastases, 20 patients $(8.5 \%, 20 / 236)$ finally showed more than 3 axillary lymph node metastases in ALND. In the group with more than 2 metastases in SLNB, 13 patients $(31.7 \%, 13 / 41)$ were diagnosed more than 3 axillary lymph node metastases in ALND. Our data support the strong correlation between high tumor grading, tumor size, lymphangiosis carcinomatosa and axillary metastatic disease.

These results highlight that in our study cohort with clinically unsuspicious axilla, a substantial number of patients would not have met the indication for intense dose-dense systemic adjuvant therapy and supraclavicular fossa radiotherapy without ALND. Furthermore, our study indicates that more than 2 positive SLNs are a strong predictor for extensive axillary lymph node metastases.

Since the German breast cancer guidelines give the option of omitting ALND in cases with clinically unsuspicious lymph node status [4], the above-mentioned tumor characteristics should be taken into account for treatment planning. Further studies are also needed to define subgroups of patients who do not profit from any axillary treatment. Interesting insights are expected from the ongoing German-Austrian INSEMA trial. Patients with 1-2 positive SLNs were randomized to SLNB alone versus ALND [24]. Adjuvant radiotherapy has also been shown to improve the outcome for patients with 1-3 lymph node metastases; the EBCTCG published evidence for the reduction of mortality and local recurrence using lymph node radiation after mastectomy and axillary surgery.

Our data highlight the role of risk factors for axillary metastatic disease that have to be taken into account for potential axillary surgery, since intense dose-dense adjuvant systemic chemotherapy has proved its effectiveness in high-risk patient subgroups [15-17]. ALND should not be considered a therapeutic operative procedure. We still think that ALND is highly relevant for adequate operative staging to indicate the optimal adjuvant therapy. This may be intense dose-dense chemotherapy or lymph node radiation. These therapies can only be indicated when ALND is performed. Our data highlight the role of ALND even with clinically unsuspicious axilla. Incorporating this approach in treatment concepts, guideline-adherent performance of ALND in breast cancer patients can thus both improve patient outcome and reduce postoperative morbidity.

In conclusion, the side effects of ALND causing increased patient morbidity are well known. We provide evidence that ALND after positive SLNB is highly relevant as a staging procedure even with clinically unsuspicious axilla. The number of excised lymph nodes should not exceed the number needed for adjuvant therapy indication, since the identification of the high-risk patient subgroup with more than 3 positive axillary lymph nodes can significantly increase the disease-free and overall survival rates. Both aspects should be taken into account using the SLNB technique.

\section{Acknowledgement}

We would like to thank Prof. Holger Kaesemann (Hanau) and Dr. Elke Schulmeyer (Gelnhausen) for the approval of the data analysis at the MainKinzig-Area Hospitals.

\section{Disclosure Statement}

All authors declare that they have no conflict of interests.

\section{References}

1 Deutsche Krebsgesellschaft: Basis-Informationen Brustkrebs/Mammakarzinom. 2015. http://www.krebsgesellschaft.de/basis-informationen-krebs/krebsarten/ brustkrebs.html (last accessed September 12, 2017).

2 Lakhani SR, Ellis IO, Schnitt SJ, et al.: WHO Classification of Tumours of the Breast, vol. 4 - IARC WHO Classification of Tumours, No. 4. Lyon, International Agency for Research on Cancer, 2012

3 Gondos A, Jansen L, Heil J, et al.: Time trends in axilla management among early breast cancer patients: persisting major variation in clinical practice across European centers. Acta Oncol 2016;55:712-719.
4 Arbeitsgemeinschaft der Wissenschaftlichen Medizinischen Fachgesellschaften e.V., Deutschen Krebsgesellschaft e.V., Deutschen Krebshilfe: S3-Leitlinie Mammakarzinom. w2012. http://www. leitlinienprogrammonkologie.de/leitlinien/mammakarzinom/ (last accessed September 12, 2017).

5 Giuliano AE, Hunt KK, Ballman KV, et al.: Axillary dissection vs no axillary dissection in women with invasive breast cancer and sentinel node metastasis: a randomized clinical trial. JAMA 2011;305:569-575.
6 Fisher B: Der Einfluß aktueller Ergebnisse klinischer NSABP Studien auf Paradigmen zur Behandlung und Prävention des primären invasiven Mammakarzinoms, in Untch M, Sittek H, Bauerfeind I, et al. (eds): Diagnostik und Therapie des Mammakarzinoms. State of Art 2004, 4th ed. Munich, Zuckschwerdt,2004, pp 480-481.

7 Bonneau C, Hequet D, Estevez JP, et al.: Impact of axillary dissection in women with invasive breast cancer who do not fit the Z0011 ACOSOG trial because of three or more metastatic sentinel lymph nodes. Eur J Surg Oncol 2015;41:998-1004. 
8 Rudenstam CM, Zahrieh D, Forbes JF, et al.: Randomized trial comparing axillary clearance versus no axillary clearance in older patients with breast cancer: first results of International Breast Cancer Study Group Trial 10-93. J Clin Oncol 2006;24:337-344.

9 Orr RK: The impact of prophylactic axillary node dissection on breast cancer survival - a Bayesian metaanalysis. Ann Surg Oncol 1999;6:109-116.

10 Schrenk P, Rieger R, Shamiyeh A, Wayand W: Morbidity following sentinel lymph node biopsy versus axillary lymph node dissection for patients with breast carcinoma. Cancer 2000;88:608-614.

11 Del Bianco P, Zavagno G, Burelli P, et al.: Morbidity comparison of sentinel lymph node biopsy versus conventional axillary lymph node dissection for breast cancer patients: results of the sentinella-GIVOM Italian randomised clinical trial. Eur J Surg Oncol 2008;34: 508-513.

12 Burak WE, Hollenbeck ST, Zervos EE, et al.: Sentine lymph node biopsy results in less postoperative morbidity compared with axillary lymph node dissection for breast cancer. Am J Surg 2002;183:23-27.

13 Schulze T, Mucke J, Markwardt J, et al.: Long-term morbidity of patients with early breast cancer after sentinel lymph node biopsy compared to axillary lymph node dissection. J Surg Oncol 2006;93:109-119.

14 Al-Hajj M, Clarke MF: Self-renewal and solid tumor stem cells. Oncogene 2004;23:7274-7282.

15 Moebus V, Jackisch C, Lueck HJ, et al.: Intense dosedense sequential chemotherapy with epirubicin, paclitaxel, and cyclophosphamide compared with conventionally scheduled chemotherapy in high-risk primary breast cancer: mature results of an AGO phase III study. J Clin Oncol 2010;28:2874-2880.

16 Untch M, Moebus V, Kuhn W, et al.: Intensive dosedense compared with conventionally scheduled preoperative chemotherapy for high-risk primary breast cancer. J Clin Oncol 2009;27:2938-2945.
17 Citron ML, Berry DA, Cirrincione C, et al.: Randomized trial of dose-dense versus conventionally scheduled and sequential versus concurrent combination chemotherapy as postoperative adjuvant treatment of node-positive primary breast cancer: first report of Intergroup Trial C9741/Cancer and Leukemia Group B Trial 9741. J Clin Oncol 2003;21:1431-1439.

18 National Collaborating Centre for Cancer: Early and Locally Advanced Breast Cancer: Diagnosis and Treatment. NICE Clinical Guidelines 80. Cardiff, National Collaborating Centre for Cancer, 2009. https://www. ncbi.nlm.nih.gov/books/n/nicecg80/pdf/ (last accessed September 12, 2017).

19 Viani GA, Godoi da Silva LB, Viana BS: Patients with N1 breast cancer: Who could benefit from supraclavicular fossa radiotherapy? Breast 2014;23:749-753.

20 McGale P, Taylor C, Correa C, et al.: Effect of radiotherapy after mastectomy and axillary surgery on 10-year recurrence and 20-year breast cancer mortality: metaanalysis of individual patient data for 8135 women in 22 randomised trials. Lancet 2014;383:2127-2135.

21 Heuser T, Rink T, Weller E, et al.: Impact of the axillary nodal status on sentinel node mapping in breast cancer and its relevance for technical proceeding. Breast Cancer Res Treat 2001;67:125-132.

22 Rink T, Heuser T, Fitz H, et al.: Results of a standardized protocol for sentinel node imaging in breast cancer with Tc-99m labeled nanocolloidal albumin. Nuklearmedizin 2001;40:80-85.

23 Rink T, Heuser T, Fitz H, et al.: Lymphoscintigraphic sentinel node imaging and gamma probe detection in breast cancer with Tc-99m nanocolloidal albumin: results of an optimized protocol. Clin Nucl Med 2001;26: 293-298.

24 Reimer T, Hartmann S, Stachs A, Gerber B: Local treatment of the axilla in early breast cancer: Concepts from the national surgical adjuvant breast and bowel project B-04 to the planned intergroup sentinel mamma trial. Breast Care (Basel) 2014;9:87-95.

25 Bao J, Donovan C, Chung A, Giuliano AE: The staging value of sentinel lymph node biopsy for breast cancer: translating pathologic findings to clinical practice. Chin Clin Oncol 2016;5:36.

26 Janni W, Kuhn T, Schwentner L, et al.: Sentinel node biopsy and axillary dissection in breast cancer: the evidence and its limits. Dtsch Arztebl Int 2014;111:244249.

27 Ibrahim-Zada I, Grant CS, Glazebrook KN, Boughey JC: Preoperative axillary ultrasound in breast cancer: safely avoiding frozen section of sentinel lymph nodes in breast-conserving surgery. J Am Coll Surg 2013;217: 7-15; discussion 15-16.

28 Dengel LT, Van Zee KJ, King TA, et al.: Axillary dissection can be avoided in the majority of clinically node-negative patients undergoing breast-conserving therapy. Ann Surg Oncol 2014;21:22-27.

29 Stachs A, Gode K, Hartmann S, et al.: Accuracy of axillary ultrasound in preoperative nodal staging of breast cancer - size of metastases as limiting factor. Springerplus 2013;2:350.

30 Wang Z, Wu LC, Chen JQ: Sentinel lymph node biopsy compared with axillary lymph node dissection in early breast cancer: a meta-analysis. Breast Cancer Res Treat 2011;129:675-689.

31 Zetterlund LH, Frisell J, Zouzos A, et al.: Swedish prospective multicenter trial evaluating sentinel lymph node biopsy after neoadjuvant systemic therapy in clinically node-positive breast cancer. Breast Cancer Res Treat 2017;163:103-110

32 Pesek S, Ashikaga T, Krag LE, Krag D: The false-negative rate of sentinel node biopsy in patients with breast cancer: a meta-analysis. World J Surg 2012;36:22392251. 